\title{
Quetiapine, Misuse and Dependency: A Case-Series of Questions to a Norwegian Network of Drug Information Centers
}

\author{
Jan Anker Jahnsen (10 ${ }^{1-3}$ \\ Sofia Frost Widnes ${ }^{1,2}$ \\ Jan Schjøtt ${ }^{1-3}$
}

\begin{abstract}
'Regional Medicines Information and Pharmacovigilance Centre (RELIS Vest), Haukeland University Hospital, Bergen, Norway; ${ }^{2}$ Department of Clinical Biochemistry and Pharmacology, Haukeland University Hospital, Bergen, Norway; ${ }^{3}$ Department of Clinical Science, Faculty of Medicine and Dentistry, University of Bergen, Bergen, Norway
\end{abstract}

Purpose: The second-generation antipsychotic quetiapine has been associated with misuse and dependency. We aimed to review questions to the Norwegian network of drug information centers concerning this potential drug safety problem.

Methods: We conducted a Boolean search in the database of the Regional Medicines Information and Pharmacovigilance Centres in Norway (RELIS) combining the indexed categories "quetiapine" and "adverse drug reaction" with the text words "misuse" or "dependency". Question-answer pairs (Q/As) in the full-text, searchable RELIS database were defined as cases. Cases were analyzed for drug safety issues linked to use of quetiapine, including off-label use, polypharmacy and other patient risk factors.

Results: The search resulted in 54 cases. Forty-six cases $(85 \%)$ were patient-related, and a majority came from physicians working in hospitals. Twenty-nine cases (54\%) concerned patients with a history of addiction, 14 cases (26\%) had polypharmacy, and off-label use of quetiapine for insomnia was identified in 14 of the cases $(26 \%)$. Only three of the cases included a specific question about patient dependency of quetiapine, and these cases were all associated with insomnia.

Conclusion: We conclude that our case series from the Norwegian network of drug information centres reflects that quetiapine frequently involves clinical narratives of a history of addiction, polypharmacy or insomnia (off-label use). However, the case series did not reveal new information about the drug's addictive potential.

Keywords: off-label, insomnia, drug safety, addiction, abuse

\section{Introduction}

Several reports have associated the second-generation antipsychotic (SGA) quetiapine with misuse and dependency. ${ }^{1-4}$ The evidence is mainly based on observational data, such as case reports, case series and database studies, including data from, for example, emergency departments, prisons and substance abuse treatment. ${ }^{1-6}$ The Drug Abuse Warning Network (DAWN) reports that emergency department visits involving misuse and abuse of quetiapine increased $90 \%$ of from 2005 to 2011 among the US general population. ${ }^{1}$ Historically, the terms abuse and misuse have been used interchangeably in the literature with varying definitions. ${ }^{7}$ We will in this manuscript use the term misuse, as recommended by the National Institute on Drug Abuse (NIH). ${ }^{8}$ In the aftermath of the DAWN report, both the European and American databases for spontaneous adverse events have been analyzed for data regarding misuse of quetiapine. $^{3,6}$ The European and American studies both concluded that quetiapine, in
Correspondence: Jan Anker Jahnsen Pharmacovigilance Centre (RELIS Vest) Haukeland University Hospital, Bergen, Norway

Tel +47-48I8207l

Email jan.anker.jahnsen@helse-bergen.no 
comparison with other SGAs, might possess a significantly higher potential for misuse. ${ }^{3,6}$ A retrospective study of national poison centers data in the US from 2005 to 2011, found 3116 cases regarding misuse or abuse of quetiapine, where $76 \%$ of the patients were treated in the emergency department and/or received medical admission. ${ }^{9}$ While previous data from single cases and prisons indicated that the population prone to misuse of quetiapine is older with a previous substance misuse history, the data from US poison centres showed median age for abuse at 17 years and median age for misuse at 26 years. ${ }^{9}$ A different study, also analyzing data from US poison centers, reports that the patients intentionally misuse quetiapine attempting to gain a favorable pharmacological effect. ${ }^{10}$ The National Poison Data System in the US defines misuse and abuse as two separate events. ${ }^{7}$

Several factors have been proposed to explain the association, including attractive sedative and anxiolytic effects, together with a more favorable safety profile than benzodiazepines, and increased availability through street value and diversion. ${ }^{4}$ The combination of histaminic blockade with comparatively mild action at dopamine receptors are suggested as the pharmacological reason for potential misuse of quetiapine. ${ }^{11}$ A recent review suggested that quetiapine's popularity in special populations is because the unpleasant or unwanted effects of addiction substances are soothed by quetiapine. ${ }^{12}$ However, it is controversial whether quetiapine and other SGAs have addictive potential by producing euphoria and other desirable effects given the dopamine hypothesis of addiction. ${ }^{5}$ Furthermore, the potential for misuse and dependency is difficult to define based on off-label prescribing and the observation that populations at higher risk of misuse are associated with forensic, psychiatric and addiction treatment settings. ${ }^{5}$

Historically, pharmacological treatment of insomnia has been a source of misuse of the prescribed drug. ${ }^{13}$ From barbiturates, to benzodiazepines, to Z-drugs, each new class of drug has been introduced with the promise of effective treatment with reduced safety risks. ${ }^{14,15}$ Despite extensive studies before gaining market authorization, problematic safety issues, including misuse and dependency, have been discovered post-marketing for drugs that effectively treat insomnia. ${ }^{14,15}$ Consequently, there is in clinical practice a need for effective pharmacological treatment of insomnia without the risk of misuse, leading to extensive use of offlabel pharmacological treatment. In Norway, quetiapine is approved for treatment of schizophrenia and bipolar disorder and as supplementary treatment for depression, while the pattern of quetiapine dispensing most likely reflects predominant off-label use, possibly explained by increased use against insomnia. ${ }^{16,17}$ In this context, the lack of systematic studies of the safety profile of quetiapine when used for insomnia is worrying.

Polypharmacy has the potential to adversely affect patient safety by increasing side effects and drug interactions while reducing adherence. Researchers have expressed concern over the widespread off-label prescribing and polypharmacy among young patients. ${ }^{18}$ Both patients with a substance abuse disorder and psychiatric patients are prone to polypharmacy. ${ }^{19}$ Due to the nature of psychiatric illness, polypharmacy can be necessary for the individual patient. ${ }^{20}$ This can be termed as appropriate polypharmacy, but in order to identify either inappropriate or appropriate polypharmacy, it is necessary to evaluate each individual case. ${ }^{21}$ As many studies are lacking the necessary clinical information to perform such evaluations, the most used definitions of polypharmacy is the use of five or more drugs. ${ }^{21}$

The possibility to process unstructured textual data, from for example suspected adverse drug reaction (ADR) reports, medical literature, electronic health records, and social media, is of current interest in pharmacovigilance. ${ }^{22}$ A disproportionality analysis to identify drug safety signals in a database shared by the Regional Medicines Information and Pharmacovigilance Centres in Norway (RELIS) was recently performed. In this study, the data mining algorithm (reporting odds ratio; ROR) found that quetiapine was associated with the text words misuse or dependency twice as common as would be expected if there was no statistical association between the drug and the event. ${ }^{23}$

This study aimed to examine this association by reviewing spontaneous questions to RELIS concerning misuse/abuse or dependency when quetiapine was prescribed, or considered for prescribing. Despite several case reports and an increasing concern in the literature, there are still many questions and few definitive answers. ${ }^{12}$ Descriptive information retrieved from clinician's queries can lead to new insight into patient characteristics and reflect the physician's attitude to this topic, while the workplace of the physician can provide information about the level of care of the patient. In addition to investigating misuse including off-label use of quetiapine for insomnia, we were particularly interested in whether or not queries suggested an addictive potential with use of the drug as an antipsychotic agent for approved indications. 


\section{Methods}

The authors work at the Regional Medicines Information and Pharmacovigilance Centres in Norway (RELIS). RELIS is funded by the Norwegian Ministry of Health and Care Services with the Norwegian Medical Agency as grant administrator, and is independent of the pharmaceutical industry. ${ }^{24}$ RELIS provide decision support to health care professionals in all four Norwegian health regions free of charge, and handle spontaneous ADR reports from health care professionals. ${ }^{25}$ The centers are associated with clinical pharmacology units at university hospitals, and the staff includes pharmacists and physicians with expertise in searching for and critically evaluating literature. ${ }^{24}$

\section{The RELIS Database}

Questions to RELIS are clinical narratives submitted in a free form text field. RELIS store indexed questionanswer pairs $(\mathrm{Q} / \mathrm{As})$ in a full-text, searchable database. The $\mathrm{Q} / \mathrm{As}$ are indexed with profession (eg, physician, pharmacist) and workplace (eg, general practice, hospital or specialist practice) for each inquirer. The RELIS database, launched in 1995 , now contains more than $50,000 \mathrm{Q} /$ As. All content of the database is available to staff working at RELIS. Selected Q/As are published online as open access and are freely available to healthcare professionals. Answers considered as not having any general interest, or questions that are so specific there is a risk the patient can be identified (eg, rare syndromes), are only available for the RELIS staff. There are no personally identifiable information (eg, name or date of birth) about patients in the database. The database contains a simple search function where either an indexed drug (eg, quetiapine), an indexed category (eg, adverse effect) or a text word (eg, dependency) is entered. Simple searches may then be combined with Boolean operators (AND/OR/NOT) ${ }^{26}$

\section{Search Strategy}

We searched the RELIS database for Q/As indexed with quetiapine and the keywords "misuse" or "dependency". The Q/As in the database is in Norwegian, and "misuse" and "dependency" are English translations of the terms used. Notice that in Norwegian, misuse can also be interpreted as abuse. The search was performed in February 2020, including all Q/As in the database from its inception in 1995. Quetiapine first received approval in the USA in 1997 and in Norway in 2003. ${ }^{12,16}$ Indexed searches were "quetiapine" and "adverse effect", while text word searches were "misuse" and "dependency". Several synonyms and combinations of Norwegian text words related to misuse, abuse, dependency and drugseeking behavior were tested to identify all relevant $\mathrm{Q} /$ As in the database. The final search string was ((quetiapine (indexed) AND adverse effect (indexed)) AND (misuse (text word) OR dependency (text word))). Figure 1 shows the search strategy in the present study.

\section{Analysis}

The questions $(\mathrm{Q})$ in the identified $\mathrm{Q} / \mathrm{As}$ were defined as cases. The text in each $\mathrm{Q}$ was manually examined with agreement among the authors with regard to type of question (patient-related or general) and patient characteristics, as presented in Table 1. The accompanying answers (A) were not analyzed. We define patient-related question as concerning pharmacotherapy in a particular patient, and general question as concerning pharmacotherapy in a patient population. Available patient characteristics are dependent on what the clinician has chosen to include in the free-form text field.

Off-label use of quetiapine regards in our experience often insomnia, and cases were reviewed for description of such use. Quetiapine is also often involved in psychotropic polypharmacy, and we defined polypharmacy as either concurrent use of three or more psychotropic drugs or five or more drugs of any type. Definition of other patient characteristics was based on the narratives that usually contain the clinical background for a question (eg, "I have a bipolar patient with anxiety and insomnia, and a history of addiction").

\section{Results}

The Boolean search resulted in 54 cases among $50,328 \mathrm{Q} /$ As in total (Figure 1). Table 1 shows the result of the manual text analysis of the 54 cases. Forty-six cases (85\%) were related to specific patients, meaning it was obvious from the narrative in the case that the question concerned a specific patient. All 54 cases were from either physician $(89 \%)$ or pharmacist $(11 \%)$, with the majority emanating from physicians working in hospital or specialist practice (78\%). In 29 cases (54\%), the narrative described a patient with a history of addiction, while polypharmacy was included in 14 cases (26\%). In five cases (9\%) clinicians were enquiring about quetiapine's addictive potential. Among them were three patient-specific cases (6\%) associated with dependency, where two were associated with 


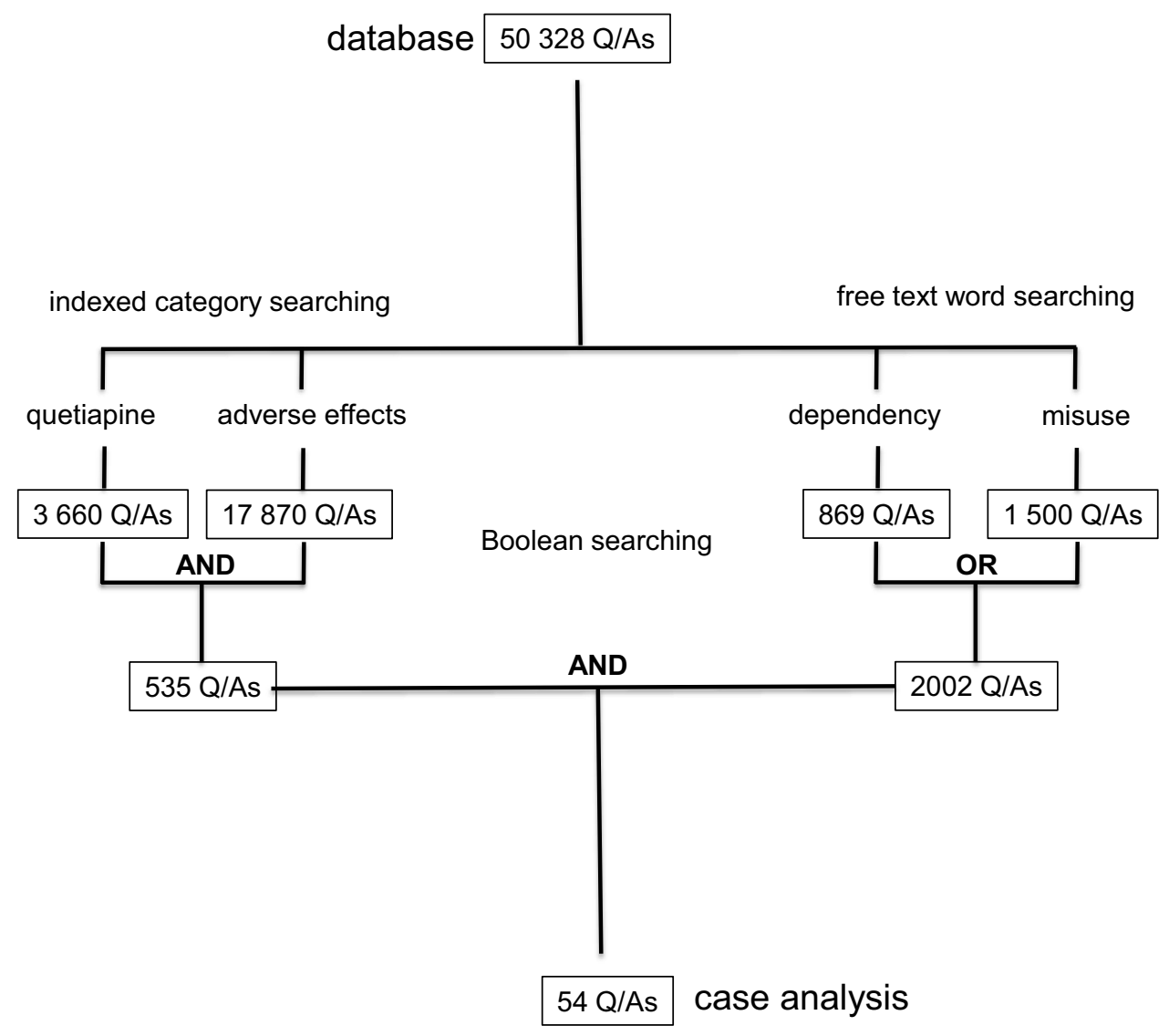

Figure I Search strategy in the database of the Regional Medicines Information and Pharmacovigilance Centres in Norway (RELIS). ${ }^{26}$ The search was based on question (Q)answering (A) pairs from March 1995 to February 2020 in the indexed, full-text, searchable RELIS database. Questions (Qs) in the Q/As were defined as cases.

off-label use of quetiapine for insomnia, while the third case related insomnia with tapering of quetiapine. Two additional cases (4\%) were from physicians enquiring about quetiapine's addictive potential based on their clinical practice. None of the five cases specified that it regarded use for an approved indication of the drug (Table 2).

\section{Discussion}

In this study, we found no cases regarding misuse or dependency among patients with regular prescribing of quetiapine for the approved indications of the drug in Norway. In other words, there were no cases, or experience from own practice, that described patients with a history of psychosis that showed any sign of addiction to the drug in therapeutic doses. The majority of cases described a history of addiction, off-label use for insomnia and psychotropic polypharmacy. All of these patient characteristics may be defined as potential risk factors to misuse of the drug. There were only a few cases where the addictive potential of quetiapine specifically was asked for.
Among these, there were three patient-specific cases, that all included subjective patient reporting. In such cases, many patients, including those without a psychiatric disease, may however experience tolerance or drug withdrawal reactions that do not qualify to a diagnosis of addiction according to the International Classification of Diseases (ICD). ${ }^{27}$

We were interested in cases reflecting real life clinical use of quetiapine, as opposed to general questions about use of quetiapine and related drug safety issues. In more than half of the cases, a history of addiction was mentioned. Our general impression from these cases was that use of quetiapine was not seen as problematic, even with a history of addiction and prevalent off-label use for insomnia. They typically included general information about a history of addiction and current pharmacotherapy (including quetiapine) - while the specific question to RELIS concerned other aspects of the treatment. This correlates with experience from clinical practice, as quetiapine is used off-label to ease symptoms of substance use disorders, including alcohol. ${ }^{12}$ The prevalent use of 
Table I Case Analysis of Questions Regarding Misuse or Dependency

\begin{tabular}{|l|l|}
\hline Analysis & Cases N=54 (\%) \\
\hline $\begin{array}{l}\text { Type of question } \\
\text { Patient-related } \\
\text { General }\end{array}$ & $46(85)$ \\
\hline Questioner & $8(15)$ \\
Physician & \\
Pharmacist & $48(89)$ \\
\hline Workplace & $6(11)$ \\
Hospital or specialist practice & \\
General practice & $42(78)$ \\
Pharmacy & $6(11)$ \\
\hline Patient characteristics & $6(11)$ \\
History of addiction & \\
Polypharmacy & $29(54)$ \\
Off-label use (insomnia) & $14(26)$ \\
Questions about quetiapine's addictive & $14(26)$ \\
potential in & $5(9)$ \\
An individual patient & \\
A patient population & $3(6)$ \\
\hline
\end{tabular}

Notes: "Type of question", "Questioner" and "Workplace" are indexed in the database for each case. Patient characteristics is information resulting from manual text analysis of the questions, performed by the authors.

quetiapine among patients with a history of addiction in our data, can explain why the previously mentioned disproportionality analysis from the RELIS database found that quetiapine was associated with the words misuse or dependency. ${ }^{23}$

Of the 54 cases, only five contained questions about quetiapine's addictive potential (Table 1). The five questions have been translated to English and are presented in Table 2. Although few, four of the five cases were registered in the last five years of the study period, in correlation with increased awareness of this issue in national and international literature. ${ }^{12,17,28,29}$ Only three cases regarded potential misuse or dependency in individual patients (Table 1). All of these were subjective patient reports, implying that the physician did not objectively suspect any misuse or dependency, and it was information from the patient that prompted the physician to ask RELIS. Two of the cases concerned off-label treatment with low-dose quetiapine for insomnia, which carries a high risk for misuse. ${ }^{12}$ Patients with a quetiapine addiction usually start with low doses and subsequently increase them, and have in most cases psychiatric comorbidities or a history of substance abuse disorder. ${ }^{12}$ Off-label treatment of insomnia with quetiapine has become prevalent in later years, ${ }^{12,16}$ and if quetiapine has a true addictive potential we should expect an increase in ADR reports associated with the drug for this indication. Analysis of ADR reports in Europe and the USA has shown a higher potential for misuse than other SGAs. ${ }^{3,6}$ The European study covers the years 2004-2016, while the American study analyzes the years 2015-2017. ${ }^{3,6}$ Considering that attention to misuse of quetiapine has increased significantly after $2015,{ }^{12}$ it will be interesting to see if this is reflected in ADR reporting in the future. A speculation is that some of the ADR reports in the above studies are based on similar descriptions observed in this study, and that the true misuse potential of quetiapine is ambiguous. The third case reported recent insomnia after a slow tapering of $400 \mathrm{mg}$ quetiapine (Table 2). Withdrawal of quetiapine can lead to "discontinuation" symptoms, ${ }^{12}$ although tapering over several months would be expected to negate such reactions. The cases containing general questions regarding quetiapine and the risk of misuse and dependency were from a psychiatrist experienced in treating substance dependency, and from a general practitioner (GP) working in a prison, respectively (Table 2). These two cases are the first registered questions about quetiapine misuse to RELIS, which is in agreement with these populations being susceptible to misusing quetiapine. ${ }^{12}$

Questions to RELIS are spontaneous, and do not necessarily represent drug problems perceived by the general population of health care professionals. However, in comparison to spontaneous ADR-reports, a question to RELIS is in the context of clinical decision support, and as such a more deliberate action. Drug safety problems, in particular off-label prescribing of quetiapine for insomnia is currently discussed in Norway. ${ }^{16,17,28,29}$ In our experience, such publicity usually motivates frequent questions to RELIS about a drug. Therefore, we were surprised that so few queries concerned misuse or dependency of quetiapine. Furthermore, in our cases, specialists did not consider quetiapine to necessarily be a problem, but the associated discussion about misuse and potential addiction linked to the drug was a concern among GPs. If treatment with quetiapine is off-label and not clearly communicated to GPs, it could lead to misunderstandings and concerns about the drug's safety profile.

The queries to RELIS are submitted as free-form text, and usually contain practical, descriptive information about the patient (eg, history of alcohol addiction) without specific diagnoses. For example, insomnia was the main indication 
Table 2 Quotes from Questions About Quetiapine's Addictive Potential

\begin{tabular}{|l|l|l|l|}
\hline & Who & When & What \\
\hline I & General practitioner & September 2016 & $\begin{array}{l}\text { A patient uses } 50 \mathrm{mg} \text { quetiapine for insomnia. The patient reports problems with } \\
\text { dependency, and cannot sleep without quetiapine. The physician asks if dependency is } \\
\text { a known risk associated with quetiapine. }\end{array}$ \\
\hline 2 & $\begin{array}{l}\text { Specialist in } \\
\text { psychiatry }\end{array}$ & January 2017 & $\begin{array}{l}\text { A patient reports recent insomnia after tapering and withdrawal of quetiapine 400 mg. The } \\
\text { tapering was performed over } 2 \text { months with concomitant significant insomnia. The } \\
\text { physician asks if this withdrawal reaction is a known effect of quetiapine, and if it could be } \\
\text { linked to dependency. }\end{array}$ \\
\hline 3 & $\begin{array}{l}\text { General practitioner } \\
\text { Specialist in }\end{array}$ & November 2018 & $\begin{array}{l}\text { A physician asks if use of quetiapine in a low dose for insomnia can result in dependency, } \\
\text { based on a report from a patient. }\end{array}$ \\
\hline 5 & $\begin{array}{l}\text { Prison physician/ } \\
\text { general practitioner }\end{array}$ & January 20I5 & $\begin{array}{l}\text { A psychiatrist who is experienced in treating substance dependency has several patients } \\
\text { requesting quetiapine, in her experience due to its effects on racing thoughts, depressive } \\
\text { thoughts and insomnia. The psychiatrist asks if it is still correct to think of quetiapine as } \\
\text { a drug that is not intoxicating. }\end{array}$ \\
\hline 5 & $\begin{array}{l}\text { addiction. Demand for quetiapine has increased among inmates, often among patients with } \\
\text { a history of addiction, who specifically ask for dosages of } 200 \text { mg or above. }\end{array}$ \\
\hline
\end{tabular}

Notes: The five cases asking about quetiapine's addictive potential. Case I-3 concern individual patients, while case 4 and 5 are general questions regarding specific patient populations. The texts are translated from Norwegian, with minor revisions to increase readability.

for use in 14 cases (26\%), but we cannot distinguish primary insomnia from other forms of insomnia due to lack of precision in the clinical narratives. This also means that we only know of concomitant medications when it is contained in the narrative, and in our experience clinicians often only report those medications they consider relevant for their question.

The drug safety issues associated with quetiapine appear to be similar to those described for gabapentine and pregabaline, where particular subpopulations are at risk of misuse. ${ }^{25,30}$ We have previously shown that our access to clinical narratives and ADR reports is of value concerning suspicion of misuse of prescribed drugs like pregabalin. ${ }^{25}$ Thus, studies like the present can provide complementary knowledge to observational registry studies. Off-label prescribing of quetiapine, which is prevalent in Norway, ${ }^{16,17}$ also involves a risk of lack of effect. With off-label use, dose escalation does not represent the initial step of dependency. Thus, ours and other observations show that the epidemiology of misuse of quetiapine needs further appropriate assessment, focusing on improved understanding of its addictive liability in the context of real life.

\section{Conclusion}

We conclude that our case series from the Norwegian network of drug information centers reflects that quetiapine frequently involve clinical narratives of a history of addiction, polypharmacy or insomnia (off-label use). However, the case series did not reveal new information about the drug's addictive potential.

\section{Disclosure}

The authors report no conflicts of interest in this work. There was no external funding of this study. The data accessed from RELIS is freely available to staff at RELIS, and no review or approval was required for this research. No further data, besides what is included in the manuscript, will be shared.

\section{References}

1. Mattson ME, Albright VA, Yoon J, Council CL. Emergency department visits involving misuse and abuse of the antipsychotic quetiapine: results from the drug abuse warning network (DAWN). Subst Abuse. 2015;9:39-46. doi:10.4137/SART.S22233

2. Lee J, Pilgrim J, Gerostamoulos D, Robinson J, Wong A. Increasing rates of quetiapine overdose, misuse, and mortality in Victoria, Australia. Drug Alcohol Depend. 2018;187:95-99. doi:10.1016/j. drugalcdep.2018.03.002

3. Chiappini S, Schifano F. Is there a potential of misuse for quetiapine? Literature review and analysis of the European Medicines Agency/ European Medicines Agency adverse drug reactions' database. J Clin $\begin{array}{lll}\text { Psychopharmacol. 2018;38(1):72-79. doi:10.1097/ } & \end{array}$ JCP.0000000000000814

4. Kim S, Lee G, Kim E, Jung H, Chang J. Quetiapine misuse and abuse: is it an atypical paradigm of drug seeking behavior? J Res Pharm Pract. 2017;6(1):12-15. doi:10.4103/2279-042X.200987 
5. Montebello ME, Brett J. Misuse and associated harms of quetiapine and other atypical antipsychotics. Curr Top Behav Neurosci. 2017;34:125-139. doi:10.1007/7854_2015_424

6. Evoy KE, Teng C, Encarnacion VG, et al. Comparison of quetiapine abuse and misuse reports to the FDA adverse event reporting system with other second-generation antipsychotics. Subst Abuse. 2019;13:1178221819844205. doi:10.1177/1178221819844205

7. Smith SM, Dart RC, Katz NP, et al. Classification and definition of misuse, abuse, and related events in clinical trials: ACTTION systematic review and recommendations. Pain. 2013;154 (11):2287-2296. doi:10.1016/j.pain.2013.05.053

8. NIDA. The science of drug use and addiction: the basics. national institute on drug abuse website; June 25, 2020. Available from: https://www.drugabuse.gov/publications/media-guide/science-druguse-addiction-basics. Accessed April 30, 2021.

9. Klein-Schwartz W, Schwartz EK, Anderson BD. Evaluation of quetiapine abuse and misuse reported to poison centers. J Addict Med. 2014;8(3):195-198. doi:10.1097/ADM.0000000000000020

10. Klein L, Bangh S, Cole JB. Intentional recreational abuse of quetiapine compared to other second-generation antipsychotics. West J Emerg Med. 2017;18(2):243-250. doi:10.5811/ westjem.2016.10.32322

11. Fischer BA, Boggs DL. The role of antihistaminic effects in the misuse of quetiapine: a case report and review of the literature. Neurosci Biobehav Rev. 2010;34(4):555-558. doi:10.1016/j. neubiorev.2009.11.003

12. Vento AE, Kotzalidis GD, Cacciotti M, et al. Quetiapine abuse fourteen years later: where are we now? A systematic review. Subst Use Misuse. 2020;55(2):304-313. doi:10.1080/10826084.2019.1668013

13. Atkin T, Comai S, Gobbi G. Drugs for insomnia beyond benzodiazepines: pharmacology, clinical applications, and discovery. Pharmacol Rev. 2018;70(2):197-245. doi:10.1124/pr.117.014381

14. Wick JY. The history of benzodiazepines. Consult Pharm. 2013;28 (9):538-548. doi:10.4140/TCP.n.2013.538

15. Schifano F, Chiappini S, Corkery JM, Guirguis A. An insight into Z-drug abuse and dependence: an examination of reports to the European Medicines Agency Database of suspected adverse drug reactions. Int $J$ Neuropsychopharmacol. 2019;22(4):270-277. doi:10.1093/ijnp/pyz007

16. Gjerden P, Bramness JG, Tvete IF, Slørdal L. The antipsychotic agent quetiapine is increasingly not used as such: dispensed prescriptions in Norway 2004-2015. Eur J Clin Pharmacol. 2017;73(9):1173-1179. doi:10.1007/s00228-017-2281-8

17. Debernard KAB, Frost J, Roland PH. Quetiapine is not a sleeping pill. Tidsskr nor Laegeforen. 2019;139(13). English, Norwegian. doi: 10.4045/tidsskr.19.0205. PMID: 31556541
18. Kearns MA, Hawley KM. Predictors of polypharmacy and off-label prescribing of psychotropic medications: a national survey of child and adolescent psychiatrists. J Psychiatr Pract. 2014;20(6):438-447. doi:10.1097/01.pra.0000456592.20622.45

19. Reuss CF, Hasselstrøm JB, Linnet K, et al. Increased risk of fatal intoxication and polypharmacy among psychiatric patients at death. J Forensic Sci. 2021;66(1):255-264. doi:10.1111/1556-4029.14586

20. Baandrup L. Polypharmacy in schizophrenia. Basic Clin Pharmacol Toxicol. 2020;126(3):183-192. doi:10.1111/bcpt.13384

21. Masnoon N, Shakib S, Kalisch-Ellett L, Caughey GE. What is polypharmacy? A systematic review of definitions. BMC Geriatr. 2017;17 (1):230. doi:10.1186/s12877-017-0621-2

22. Ventola CL. Big data and pharmacovigilance: data mining for adverse drug events and interactions. $P$ T. 2018;43(6):340-351.

23. Stokes CL, Schjøtt J. Disproportionality analysis for identification of drug safety signals in a database shared by the Norwegian network of drug information centres (RELIS). Eur J Clin Pharmacol. 2019;75 (10):1469-1470. doi:10.1007/s00228-019-02717-x

24. Schjøtt J. Benefits of a national network of drug information centres: RELIS. Eur J Clin Pharmacol. 2017;73(1):125-126. doi:10.1007/ s00228-016-2129-7

25. Schjøtt J, Bergman J. Joint medicine-information and pharmacovigilance services could improve detection and communication about drug-safety problems. Drug Healthc Patient Saf. 2014;6:89-92. doi:10.2147/DHPS.S63680

26. Schjøtt J, Reppe LA, Roland PD, Westergren T. A question-answer pair (QAP) database integrated with websites to answer complex questions submitted to the Regional Medicines Information and Pharmacovigilance Centres in Norway (RELIS): a descriptive study. BMJ Open. 2012;2(2):e000642. doi:10.1136/bmjopen-2011-000642

27. World Health Organization. ICD-10 online versions. F10-F19 Mental and behavioural disorders due to psychoactive substance use. Available from: https://www.who.int/classifications/icd/icdonlinever sions/en/. Accessed April 30, 2021

28. Gjerden P, Bramness JG, Slørdal L. Kvetiapin brukes for mye [Quetiapine is used too much]. Tidsskr nor Legeforen. 2018:138. Norwegian. doi:10.4045/tidsskr.18.0535

29. Waal H, Vold JH, Skurtveit SO. Quetiapine abuse - myth or reality? Tidsskr nor Laegeforen. 2020;140(12). English, Norwegian. doi:10.4045/tidsskr.20.0059

30. Driot D, Jouanjus E, Oustric S, Dupouy J, Lapeyre-Mestre M. Patterns of gabapentin and pregabalin use and misuse: results of a population-based cohort study in France. Br J Clin Pharmacol. 2019;85(6):1260-1269. doi:10.1111/bcp.13892
Drug, Healthcare and Patient Safety

\section{Publish your work in this journal}

Drug, Healthcare and Patient Safety is an international, peer-reviewed open-access journal exploring patient safety issues in the healthcare continuum from diagnostic and screening interventions through to treatment, drug therapy and surgery. The journal is characterized by the rapid reporting of reviews, original research, clinical, epidemiological and post-marketing surveillance studies, risk management, health literacy and educational programs across all areas of healthcare delivery. The manuscript management system is completely online and includes a very quick and fair peer-review system. Visit http://www.dovepress.com/testimonials.php to read real quotes from published authors. 